\title{
ROLE OF HOST AGE AND GENOTYPE IN MURINE ENTEROTROPIC CORONAVIRUS INFECTION
}

\author{
Stephen W. Barthold and Abigail L. Smith \\ Section of Comparative Medicine \\ Yale University School of Medicine \\ 333 Cedar Street, P.O. Box 3333 \\ New Haven, Connecticut 06510
}

Enterotropic mouse hepatitis virus (MHV) is probably the most common form of MHV in contemporary laboratory mouse populations, but very little is known about its pathogenesis. In spite of a large volume of literature on MHV, most of these reports are related to the respiratory prototype strains of virus $(1,2)$. When several prototype MHV and enterotropic MHV strains were inoculated into infant mice, it became clear that different MHV strains produced two distinctly different patterns of disease: respiratory, in which virus disseminated from nasal epithelium to multiple target organs; or enterotropic, in which virus was largely restricted to intestine, with minimal dissemination (3). However, enterotropic MHV has been poorly characterized because of its fastidious and selective in vitro growth characteristics. Host age seems to significantly influence the outcome of disease in mice infected with enterotropic MHV (4-11), and host genotype may also be important in susceptibility to enterotropic MHV disease (12). These are well established factors in the pathogenesis of respiratory or polytropic MHV disease $(1,2)$. The present study systematically examined the relative susceptibility of inbred mice of different ages that have previously been shown to develop severe (BALB/cByJ) or 
mild (SJL/J) disease when inoculated oronasally with respiratory MHV-JHM (13). Pilot studies suggested that these mouse genotypes also display differential disease severity when infected with enterotropic MHV, strain Y (12).

\section{MATERIALS AND METHODS}

Mice and Virus. Pregnant, 3 week and 12 week old inbred BALB/cByJ (BALB) and SJL/J (SJL) mice were obtained from The Jackson Laboratory (Bar Harbor, ME) and pregnant outbred CrlCD1BR (CD1) mice were obtained from Charles River Laboratories (Portage, MI). Animal and cage manipulations were performed in a biological containment cabinet.

MHV-Y, isolated in NCTC 1469 cells from the intestine of a naturally infected infant mouse (7), was maintained by serial oral passage of $10 \%$ intestinal homogenates in infant CD1 mice. Inocula contained approximately 103 median CD1 infant mouse enteritis doses $\left(\mathrm{ED}_{50}\right)$.

Serology. MHV IgG antibody titers in sera were determined with a modified enzyme immunoassay (EIA) using formalin-fixed MHV-S-infected $17 \mathrm{Cl} 1$ cells as antigen, as described (14). MHV-Y has been shown to be more closely related antigenically to MHV-S than to other prototype MHV strains (7).

Experimental Design and Pathology. The course of MHV-Y infection was examined following oral inoculation of 1,3 and 12 week old BALB and SJL mice. On days 1, 2, 3, 5, 10, 20 and 30 after inoculation, tissues were collected for virus titration and histology. Virus titers in segments of glandular stomach, duodenum, ileum, cecum, ascending colon, descending colon, mesenteric lymph nodes, Peyer's patches and feces of 3 week old BALB mice were all determined, and based upon these results, virus was quantified in the ascending colons of other groups for comparison. Brain, lung, spleen, liver, cervical lymph node, glandular stomach, duodenum, ileum, cecum, ascending colon, descending colon, mesenteric lymph nodes, and Peyer's patches were also immersion-fixed in neutral buffered formalin, $\mathrm{pH}$ 7.2, paraffin-embedded, sectioned and stained with hematoxylin and eosin.

\section{RESULTS}

Virus quantification in different levels of intestine, gut-associated lymphoid tissue and feces of 3 week old BALB mice at intervals after oral inoculation revealed minimal or no infectious virus in stomach, but varying levels of infectious virus in other samples. Few 
samples were positive at 1 day after inoculation, but virus titers rose dramatically by 2 and 3 days, then declined at subsequent intervals. All Peyer's patch samples were positive on days 2 and 3, whereas mesenteric lymph nodes were less consistently infected. Virus was readily detected in feces on days 2,3 and 5 , but declined in parallel with tissues thereafter. These results also showed that virus titers were highest and most sustained (approximately $106 \mathrm{ED}_{50}$ per gram on days 2 through 5) in the ascending colon, compared to other sites.

Since ascending colon appeared to be the major target for virus replication and disease, virus was quantified in ascending colons of other mouse ages and strains. Among BALB mice, highest virus titers were found in mice inoculated at 1 week of age (106.5 $\mathrm{ED}_{50}$ per gram), with lower peak titers in 3 week old mice and lowest peak titers (105.2 $\mathrm{ED}_{50}$ per gram) in 12 week old mice. Remarkably, titers were low at 2 days in the BALB mice inoculated at 1 week of age, whereas titers were at their zenith at this interval in the older mice. In SJL mice, peak virus titers were more or less equivalent among mice of different ages and, except for mice inoculated at 1 week of age, equivalent to peak titers attained in BALB mice of corresponding age. Peak virus titers in 1 week old SJL mice were one $\log _{10}$ less compared to 1 week old BALB mice. Virus titers declined precipitously after peaking in mice of both genotypes and all ages, but a few mice had low levels of detectable virus at the 30 day interval.

Histologic examination supported the infectivity data. In BALB mice inoculated at 3 weeks of age, virtually no lesions were observed in stomach at any interval. On day 2, lesions in upper small intestine were confined to a few syncytia and necrosis of few enterocytes, particularly of $M$ cells overlying gut-associated lymphoid tissue. Syncytia were more apparent in the lower small intestine and cecum, most obvious in the ascending colon and rarely found in the descending colon. These changes were also observed on day 3 , but to a much lesser extent. Mucosal hyperplasia was apparent on day 5, with a return to normal by day 10 . Inflammation was minimal or absent. No lesions were evident on days 10,20 or 30 .

Histology was also performed on all levels of intestine at all intervals in BALB and SJL mice inoculated at 1, 3 or 12 weeks of age. As in BALB mice inoculated at 3 weeks of age, lesions in BALB and SJL mice inoculated at 1 week of age were most obvious and consistently present in the ascending colon, but also involved the ileum, cecum and, to a minimal degree, descending colon. BALB mice inoculated at 1 week of age had marked distortion of ascending colonic mucosa, due to crypt epithelial hyperplasia, syncytia and 
inflammation, with mild epithelial necrosis. There was no evidence of mucosal erosion or ulceration. Changes were most apparent on days 2 and 3, but continued with regularity on day 5 before waning at subsequent intervals. Hyperplasia was evident on days 2, 3, 5 and 10. Lymphoid syncytia were observed in mesenteric lymph nodes of most of these mice on day 5. In addition to intestinal lesions, all 1 week BALB mice examined on day 10 had diffuse necrotizing encephalitis, which was accompanied by sudden high mortality around this interval. The few surviving mice had no brain disease on days 20 or 30 . Focal hepatitis was also present in most 1 week BALB mice at day 10 , as well as sporadically at earlier intervals. These foci had minimal necrosis and no visible syncytia. No significant lesions were seen in other tissues.

In contrast to BALB mice, SJL mice inoculated at 1 week of age had remarkably milder disease. Only a few small syncytia were observed in the cecal and ascending colonic mucosa on day 3, but hyperplasia was seen on days $3,5,10$ and 20 . SJL mice did not develop lesions in other tissues, most notably liver and brain, and mesenteric lymph nodes were involved in only 1 mouse on day 3 .

Mice inoculated at 3 and 12 weeks of age developed very mild lesions, restricted largely to the ascending colon. Compared to 3 week old BALB mice (above), SJL mice inoculated at 3 and 12 weeks had a few syncytia visible in ascending colonic mucosa on day 2 , but not thereafter, and minimal evidence of compensatory mucosal hyperplasia. BALB mice inoculated at 12 weeks of age developed lesions similar in quality to those inoculated at 3 weeks of age, but slightly milder, with mild hyperplasia on day 5 .

Antibody to MHV was generally detected by day 10 in 3 and 12 week old mice and day 20 in mice inoculated at 1 week of age. Antibody titers were lowest in mice inoculated at 12 weeks of age.

\section{DISCUSSION}

Although enterotropic MHV is generally associated with disease of the neonate and infant, the present study indicates that significant virus replication occurs in mice of all ages, yet disease is minimal in older mice. In contrast, studies with respiratory strain MHV-JHM demonstrate a close parallel of disease severity with virus titers (13). In considering why the neonatal bowel is preferentially susceptible to disease, but not necessarily infection, Biggers et al. (5) suggested that this might be related to mucosal proliferative kinetics. Mucosal turnover rate is sluggish and mucosal epithelial cells are highly differentiated in the neonatal bowel, allowing damage by a rapidly 
replicating virus with minimal ability to respond. Older mice have less differentiated cells and higher mucosal turnover rates, allowing more rapid replacement of damaged mucosa, resulting in mild disease $(15,16)$. This is supported by the observation that syncytia, a hallmark of MHV infection, are located primarily in the surface mucosa in older mice, while virus damage occurs throughout the mucosal epithelium of younger mice.

The present study also demonstrates that SJL mice, regardless of age, support significant virus replication in intestinal mucosa, but have milder disease compared to BALB mice. SJL mice have been shown to lack a functional receptor for respiratory MHV-A59, resulting in marked resistance to virus infection and significantly reduced replication in target cells or tissues (17). We have shown previously that SJL mice allow little or no virus replication and develop minimal or no disease in liver and brain when inoculated with MHV-JHM at 3 or more weeks of age. When SJL mice were inoculated at 1 week of age, they developed disseminated infection, but virus replication and disease were reduced compared to BALB mice (13). Therefore, the resistance of the SJL mouse to infection and disease caused by respiratory strains of MHV appears to be agerelated, as with MHV-Y, but mediated through an entirely different mechanism than resistance to enterotropic MHV, since they allow significant MHV-Y replication at all ages .

In spite of the high degree of enterotropism displayed by MHV$Y$ used in this study, we confirmed previous observations (12) that MHV-Y disseminated to brain and liver in infant BALB mice, but not older BALB mice or SJL mice of any age. The hepatitis observed in the infant BALB mice was mild, unlike the severe necrotizing hepatitis commonly observed in susceptible mice infected with respiratory strains of MHV $(1,13)$. We have also observed mild and transient, rather than progressive, hepatitis in athymic nude mice infected experimentally with enterotropic MHV-RI (18). Virus was rarely detected in livers and lesions were restricted to intestine of mice with lethal intestinal virus of infant mice (LIVIM) (1-3), and no liver disease occurred in infant mice infected with enterotropic MHVS/CDC $(8,9)$. In contrast, enterotropic strain MHV-DVIM readily caused hepatitis with virus replication in liver (11). These differences underscore the unpredictably wide range of biological effects and relative tissue tropism of MHV strains and isolates. Clearly, the distinction between respiratory and enterotropic biotypes of $\mathrm{MHV}$ is relative, rather than absolute.

Our studies implicate the ascending colon as a key target for MHV-Y, while others $(4-6,8,9,11)$ have emphasized that the lower small intestine appeared to be the primary target for morphological 
virus damage, with less consistent involvement of cecum and infrequent involvement of colon. These differences may be MHV strain-related, or may be due to failure of others to examine the craniad (ascending) segment of colon. As in the present study, we have observed the ascending colon to be the most consistently affected segment of intestine involved with at least 3 different enterotropic strains of $\operatorname{MHV}(3,7,18)$. This is our impression of naturally-occurring cases as well. The current study reinforces the importance of examining all segments of intestine, but particularly ascending colon, for MHV diagnosis. The ascending colon may be the only level of intestine with pathognomic lesions in adult, asymptomatic mice.

\section{ACKNOWLEDGEMENTS}

This work was supported by grants RR02039 and RR04507 from the Division of Research Resources, National Institutes of Health, Bethesda, MD. The technical assistance of Deborah Beck and Deborah Winograd is appreciated.

\section{REFERENCES}

1. Barthold, S.W. 1986. In T.C. Hamm, (ed.), Complications of Viral and Mycoplasmal Infections in Rodents to Toxicology Research and Testing, pp.53-89. Hemisphere Publishing Corporation, Washington, DC.

2. Barthold, S.W. 1986. In P.N. Bhatt, et al. (eds), Viral and Mycoplasmal Infections of Laboratory Rodents: Effects on Biomedical Research, pp. 571-601. Academic Press, New York, NY.

3. Barthold, S.W. and A.L.Smith. 1984. Arch. Virol. 81:103-112.

4. Kraft, L.M. 1962. Science 137:282-283.

5. Biggers, D.C. et al. 1964. Amer. J. Pathol. 45:413-422.

6. Kraft, L.M. 1966. NCI Monograph 20:55-61.

7. Barthold, S.W. et al. 1982. Lab.Anim. Sci. 32:376-383.

8. Broderson, J.R. et al. 1976. Lab. Anim. Sci. 26:824.

9. Hierholzer, J.C. et al. 1979. Inf. Immun. 24:508-522.

10. Carthew, P. 1977. Vet. Rec.101:465.

11. Ishida, T. and K. Fujiwara. 1979. Jap. J. Exp. Med. 52:231-35.

12. Barthold, S.W. 1987. Lab. Anim. Sci. 37:36-40.

13. Barthold, S.W. and A.L. Smith. 1987. Virus Res. 7:225-239.

14. Smith, A.L. and D.F. Winograd. 1986. J. Virol. Methods 14:335-343

15. Tsuboi, K.K. et al. 1985. Biochim. Biophys. Acta 840:69-78.

16. Tsuboi, K.K. et al. 1981. Biochim. Biophys. Res. Comn. 101:645-652

17. Williams, R.K. et al. 1990. J. Virol. 64:3817-3823.

18. Barthold, S.W. et al. 1985. Lab. Anim. Sci. 35:613-618. 